\title{
免洗手消毒用酒精凝胶的制备实验设计与课程思政实践
}

李秋平 ${ }^{*}$, 朱琳, 胡敏杰, 罗利娟, 吴昊

宁波工程学院材料与化学工程学院, 浙江 宁波 315211

摘要: 免洗手消毒用酒精凝胶是一种基于增稠剂调节黏度且酒精含量为 $75 \%$ 左右的日用化学品, 属于精细化学品的 范畴, 在抗击新冠肺炎疫情的过程中发挥着重要的作用, 经此疫情, 相关产品已经成为大众熟知与消耗性物资。免 洗手消毒用酒精凝胶的基础配方简单, 成分易得, 生产过程简易又不失一定的难度, 其制备过程非常适合设计为精 细化学品实验或类似课程的教学内容。通过在应用化学及相关专业的大学实验教学中开设该实验, 既能增强学生对 精细化学品行业的专业兴趣, 又能培养学生的配方设计能力与锻炼学生的动手操作能力。同时, 该课程还蕴藏诸多 思政元素可以挖掘, 非常适合在实验教学过程中开展思政教育, 培养学生防范新冠肺炎传染病的意识以及对中国特 色社会主义道路的自信。

关键词: 精细化学品实验; 酒精凝胶; 实验设计; 课程思政

中图分类号: G64; O6

\section{Ideological and Political Education Implantation and Experimental Design of Alcohol-Based Hand Sanitizer Gel}

\author{
Qiuping Li ${ }^{*}$, Lin Zhu, Minjie Hu, Lijuan Luo, Hao Wu \\ School of Materials and Chemical Engineering, Ningbo University of Technology, Ningbo 315211, Zhejiang Province, China.
}

\begin{abstract}
Alcohol gel for hand-washing disinfection is a kind of daily chemical which has $75 \%$ alcohol. It belongs to the category of fine chemicals and is prepared by adjusting the viscosity of alcohol with a thickener. It has played an important role in the fight against the COVID-19 pandemic, and is familiar to people. The basic formula is simple and the ingredients are easy to obtain. Although the production process is simple, the experiment is still a bit difficult. Thus, the preparation process is very suitable for designing the teaching content of "Fine Chemical Laboratory" or similar courses. By setting up this experiment in applied chemistry specialty or related majors, the course can not only enhance students' professional interest in the fine chemical industry, but also cultivate theirs ability to design product formula and practical experience. At the same time, the course also contains many ideological and political elements, which is very suitable for carrying out ideological and political education in laboratory course. Through this course, we can enhance students' awareness of preventing COVID-19 infections, and strengthen their confidence to Chinese characteristic socialism.
\end{abstract}

Key Words: Fine chemical laboratory; Alcohol gels; Experimental design; Ideology and politics education

精细化学品实验是应用化学与精细化工方向非常重要的一门基础实践课程, 其教学内容主要围 绕常见精细化学品的生产原理、生产过程以及应用前景展开设计, 该课程既是相关专业学生未来从

收稿: 2020-08-03; 录用: 2020-09-09; 网络发表: 2020-09-30

“通讯作者, Email: liqiuping@nbut.edu.cn

基金资助: 宁波工程学院课程思政建设项目 
事精细化工产品研发与生产的重要理论基础, 又是培养学生动手能力与对精细化工行业专业兴趣的 重要实践课程 ${ }^{[1,2]}$ 。随着我国精细化工行业的迅猛发展, 精细化学品种类也越来越多, 据统计, 光我 国市场上能找到的精细化学品商品就高达 2 万多种, 而且这一数目还在逐年增长中 ${ }^{[3]}$ 。如何从众多的 精细化学品中挑选具有代表意义且操作性强的产品用作相关专业的实践教学, 使学生在掌握精细化 学品的设计原理与生产方法的同时, 更能深刻体会精细化学品在生产生活中的应用情况, 从而进一 步激发学生的专业学习兴趣, 这一问题就显得尤为迫切 ${ }^{[4]}$ 。

免洗手消毒用酒精凝胶是一种能快速灭活有害微生物的消毒剂, 是预防疾病的很好工具 $[5,6]$ 。将 消毒酒精做成凝胶状产品便于携带和保存, 与普通溶剂型消毒液相比, 消毒凝胶可有效防止酒精的 喷溅与滴落, 具有使用方便、消毒高效快速和免水洗环保等特点。在此严重新型冠状病毒蔓延的背 景下, 该产品可为一线的医护人员、志愿者等人群提供一定的便捷有效的保护。疫情初期, 面对免 洗手消毒用酒精凝胶等产品供不应求的问题, 许多网络视频和文章纷纷开始教授人们免洗手消毒用 酒精凝胶的制作方法, 抗击疫情, 砥砺前行。有鉴于此, 通过在精细化学品实验课程中开设免洗手 消毒用酒精凝胶的制作教学单元, 不仅能实现课程本身的教学目的, 还可通过教学过程增强学生的 卫生防护意识, 并在课程过程中植入许多思政元素, 从而达到立德树人的真正目的。像化学这样与 我们生活息息相关的学科, 是最容易挖掘出思政元素的, 通过简单的教学设计就可将 “立德树人” 的根本任务融入课程教学的各个环节, 实现全课程育人的格局 ${ }^{[7]}$ 。在大学化学实验教学过程中植入 思政教育元素, 也成了当下各高校化学专业实验课程的改革重点 ${ }^{[8]}$ 。

本实验是宁波工程学院应用化学专业精细化学品实验课程应新冠肺炎疫情这一突发事件而增设 的课程单元, 在课程设计过程中充分融入了多项思政教育元素, 实验操作简单, 内容贴近生活, 适 合在各级各类学校化学相关专业学生中普及开设。

\section{1 实验教学设计}

在条件允许的情况下, 本实验可由学生独自完成, 不需组队。要求学生在实验之前认真预习, 尤其要认真预习 “实验注意事项部分”, 实验过程中要认真记录实验现象和各成分实际用量, 实验 结束后可带走各自所生产的酒精凝胶产品。同时, 每位学生在实验结束之后应提交一份完整的实验 报告，并在查阅相关资料的基础上对实验结果与思考题进行认真思考。

\section{1 实验目的}

(1) 掌握免洗手消毒用酒精凝胶的配方设计原理;

(2) 练习并掌握免洗手消毒用酒精凝胶的一般生产过程;

(3) 熟悉酒精凝胶的消毒原理, 增强学生的传染病预防意识和防护能力。

\section{2 实验原理}

本实验制作的免洗手消毒用酒精凝胶的最主要成分是乙醇, 乙醇在其中主要起到杀菌消毒和挥 发性溶剂的作用。大量的医学实验已经证明, 乙醇含量 $75 \%$ (体积百分比)左右的酒精可以很好地渗 透进入病毒内部, 使所有病毒蛋白都能脱水、变性而凝固, 并最终杀死病毒。虽然提高乙醇浓度有 利于蛋白脱水变性, 但是过高的酒精浓度会使病毒外壳蛋白迅速变性凝固, 形成一层坚固的薄膜, 从而阻止酒精的进一步渗透, 导致无法彻底杀死病毒; 另一方面, 如果乙醇浓度过低, 虽可进入病 毒内部, 但由于其对蛋白质的渗透性较差, 也无法有效地使病毒蛋白变性 ${ }^{[9]}$ 。同时, 在相同温度下乙 醇的蒸气压是水的2-3倍, 其挥发速度远高于水分, 在几番搓手消毒之后能迅速挥发, 可免除洗手之 后还要擦干的不便。由于酒精具有很强的流动性和挥发性, 少量的酒精倒在手上, 稍微一搓手即会 完全挥发, 根本无法使其布满双手皮肤, 想要用普通酒精洗手消毒需消耗较大的用量才能实现完全 的消毒效果。而添加了增稠剂的酒精可在一定程度上降低其流动性与挥发性, 在搓手之时, 酒精能 轻易地以凝胶的状态涂满双手, 并在此过程中完成消毒过程, 之后随着双手的揉搓又逐渐完全挥发。

本实验制作的洗手凝胶的另一个主要成分是卡波姆, 它的主要作用就是增稠。卡波姆是一种非 
常重要的流变调节剂, 通常是由丙烯酸或丙烯酸酯与烯丙基醚经交联反应而制得的树脂材料。由于 分子结构中含有大量的羧酸基团, 卡波姆具有一定的酸性和亲水性, 可在水中显著溶胀, 但是单纯 的乙醇对卡波姆来说却是不良溶剂, 需要借助一定的有机胺才能实现这一效果。

卡波姆在溶液中可通过两种机制增稠:

(1) 通过不同卡波姆分子之间的分子链相互缠绕实现增稠效果, 由于卡波姆分子结构中含有大 量的羧基结构单元, 通过调节 $\mathrm{pH}$ 可使其羧基结构阴离子化而相互排斥, 使得初始卷曲的卡波姆分子 卷曲链舒展开, 体积增大上千倍, 增加了分子之间相互缠绕的概率, 从而大大提高卡波姆的增稠效 果。研究结果表明, 当体系的 $\mathrm{pH}$ 在6-12范围内的时候, 卡波姆的增稠效果最好。

(2) 另外, 羧基阴离子化的卡波姆分子还可通过羰基氧与多元醇分子中的羟基氢形成氢键, 并 交联成网状结构, 进而实现增稠效果。

除了上述两种主要物质外, 在生产卡波姆酒精凝胶之时, 还常用三乙醇胺调节体系的 $\mathrm{pH}$, 并加 入一定量的甘油护手和辅助增稠。当然, 还可视需求加入香精、芦荟凝胶和抗氧化剂等各种物质进 行复配, 改善产品性能, 使其更具市场竞争力。本实验所设计的酒精凝胶配方及各组分作用如表 1 所示。

表1 免洗手消毒用酒精凝胶配方

\begin{tabular}{ccc}
\hline 原料 & 含量 & 作用 \\
\hline 乙醇 & $75 \%$ & 溶剂、消毒剂 \\
卡波姆 & $0.4 \%-0.5 \%$ & 增稠剂 \\
三乙醇胺 & $2-3$ 滴 & 调节 $\mathrm{pH}$ \\
甘油 & $1 \%$ & 护手、辅助增稠 \\
玫瑰香精 & $2-3$ 滴 & 增香 \\
水 & $23 \%-24 \%$ & 溶剂 \\
\hline
\end{tabular}

本实验涉及磁力搅拌、冷凝回流等基本实验操作, 又涉及到了日用化学品的配方设计原理与基 本生产过程, 适合用作应用化学或精细化工等相关专业本专科高年级学生的专业实验。

\section{3 实验仪器与药品}

实验仪器: 分析天平、 $150 \mathrm{~mL}$ 三口烧瓶、 $150 \mathrm{~mL}$ 烧杯、恒温磁力搅拌器、恒压滴液漏斗、玻璃 棒、量筒、药匙、按压瓶、回流冷凝管、磁子、pH试纸、空心塞、称量纸。

实验药品: 卡波姆940 (上海阿拉丁生化科技股份有限公司)、三乙醇胺 $(99 \%$, 美国陶氏化学公 司)、乙醇( $95 \%$, 国药集团化学试剂有限公司)、甘油(99\%, 国药集团化学试剂有限公司)、去离子水 (自制)、玫瑰香精(杭州西湖香精香料有限公司)。

\section{4 实验步骤}

(1) 利用恒温磁力搅拌器、 $150 \mathrm{~mL}$ 三口烧瓶和回流冷凝管搭建回流冷凝反应器。

(2) 称取0.4-0.5 g卡波姆置于三口烧瓶内, 加入 $20 \mathrm{~mL}$ 去离子水, 加入磁子进行磁力搅拌。打开 冷凝水, 将反应器温度升高到 $75^{\circ} \mathrm{C}$, 在此温度下搅拌大约 $30 \mathrm{~min}$ 至卡波姆粉末完全溶胀, 体系呈透 明凝胶状, 随后将反应器温度下调到 $60{ }^{\circ} \mathrm{C}$ 。

(3) 量取 $95 \%$ 的乙醇 $79 \mathrm{~mL}$, 置于恒压滴液漏斗, 装到三口烧瓶上, 在持续回流与搅拌下, 以 2-3滴/秒的速度滴入凝胶体系中, 滴加完后再加入 $1 \mathrm{~mL}$ 甘油, 最终该体系会变成微乳白与微浑浊的 胶状溶液。

(4) 趁热将溶液转移至 $150 \mathrm{~mL}$ 烧杯, 使用三乙醇胺调节 $\mathrm{pH}$, 一滴一滴的滴加, 每次滴加完后要 充分摚拌均匀, 大约2-3滴左右即能发现体系黏稠度明显增加, 且从略微乳白浑浊状转变为完全透明 均匀的凝胶, 用 $\mathrm{pH}$ 试纸测试并记录溶液 $\mathrm{pH}$ 。 
(5) 适量滴加2-3滴玫瑰香精增香, 待搅拌均匀后将样品装入 $100 \mathrm{~mL}$ 深色挤压瓶。

（6）挤压部分产品到手上，观察产品流动性，搓揉使其涂布满双手，体验产品性能。

\section{5 实验注意事项}

(1) 卡波姆的溶胀步骤非常关键, 必须等溶液变成完全透明凝胶状才能进行后续步骤。

(2) 乙醇加入速度不可过快，且必须及时搅拌均匀，否则可能导致局部卡波姆聚沉现象。

(3) 调节 $\mathrm{pH}$ 时三乙醇胺应逐滴逐滴地滴加, 且每次滴加后应充分搅拌, 然后根据情况再决定是 否继续滴加三乙醇胺, 三乙醇胺滴加过头会使卡波姆聚沉从而导致实验彻底失败。

(4) 实验失败产品不得倒入下水道, 以免堵塞下水管道。

\section{6 实验结果}

最后应得到无色透明凝胶状且酒精含量达 $75 \%$ 左右的产品, 其外观与挤压到手上后的效果可参 考图1。体验所制备酒精凝胶的洗手效果时, 产品应在搓揉的过程中无明显颗粒感, 且经多次搓手后 迅速挥发, 无残留, 无须额外擦干为最佳。同时, 还要列出实际配方成分与用量表以供参考。

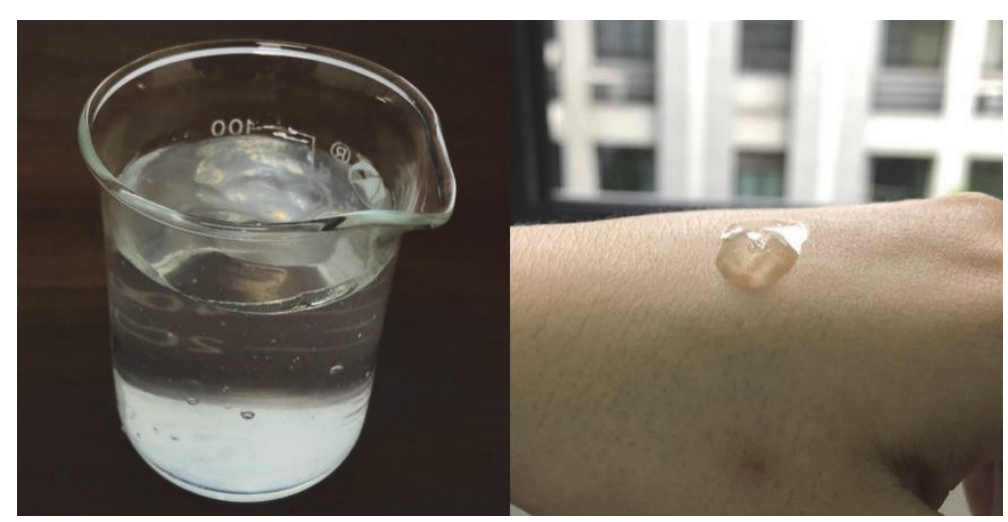

图1 酒精凝胶外观(左)与挤压至手上后的效果图(右)

\section{7 实验思考}

实验结束之后可针对实验过程中出现的各种问题与本实验未能充分实践的拓展知识设置合理的 思考题，例如:

(1) 是否可以预先将卡波姆充分溶胀, 实验之时直接使用, 而不再需要加热回流。

（2）实验过程中如果不小心加入乙醇过快导致卡波姆部分聚沉了，是否还能补救。

(3) 是否可以使用其他类型的卡波姆代替卡波姆 940 进行实验。

(4) 是否可以在配方中加入第二种消毒剂, 例如苯扎澳铵等, 以便增强消毒效果。

\section{2 课程思政设计}

作为在抗击与预防新冠肺炎中被大量使用的一种防疫物资, 制备免洗手消毒用酒精凝胶产品本 身就具有很强的现实意义, 在充分挖掘酒精凝胶产品本身特点与实验内容的基础上, 我们认为至少 可以从以下几个方面挖掘思政元素并开展课程思政育人工作:

(1) 从酒精凝胶产品的消毒应用出发, 普及酒精产品的杀菌消毒科普知识, 鼓励学生在掌握了 这些知识以后积极向亲人朋友传播相关知识, 解除许多人对酒精凝胶消毒产品的不信任心态, 提高 大家的卫生防护意识, 为国家的防疫工作贡献一份力量。

(2) 积极引导学生联想我国防疫过程中酒精凝胶和消毒药剂等各种防疫相关的精细化学品供应 充足的情况, 以及我国社会经济从新冠肺炎疫情影响中快速恢复的现实, 对比国外大部分国家各种 防疫物资紧缺和疫情防控彻底失控的状况, 激发学生爱党爱国的意识, 增强学生对中国特色社会主 义路线的自信。 
(3) 在实验过程中, 可能会遇上学生滴加乙醇过快导致卡波姆发生部分凝聚, 以及滴加过量的 三乙醇胺导致产品制备失败的情况, 此时任课教师可以积极引导学生分析失败原因和指导学生采取 补救措施。让学生体会到一时的挫折并不可怕, 关键是遇上了挫折要懂得去分析原因, 并采取相应 的措施去克服挫折与困难, 培养学生严谨细致、认真思考与不折不挠的科研精神。

(5) 还可通过这个实验引导学生正确看待精细化学品行业与工业对我国经济发展与社会发展的 重要意义, 激发学生对化学化工相关行业的专业兴趣与热情, 培养学生作为化学研究者与化学实践 者的自豪感。

\section{3 结语}

本精细化学品合成实验具有操作简单、绿色环保、成功率高等特点, 且具有强烈的现实意义和 社会意义, 既可作为生动有趣的大学化学实验, 激发学生的专业学习兴趣, 又可作为一堂课程思政 深度融合的专业课程。课程设计包含了实用性与思政性的双重教学改革目标, 因此非常适合用于应 用化学或精细化工等相关专业的本专科学生的实验教学工作。

\section{参 考 文 献}

[1] 朱敏, 田少鹏, 王登武. 课程教育研究, 2020, No. 5, 174.

[2] 田建文, 张小勇, 严兆华. 江西化工, 2020, No. 2, 1 .

[3] 王敏, 李峰. 云南化工, 2018, 45 (10), 21.

[4] 武荣兰, 刘罡. 信息记录材料, 2018, 19 (5), 150.

[5] 俞秋华, 付喜梅, 刘莉莉, 杨宁. 生物化工, 2019, 5 (2), 66.

[6] 王钰荣, 赵丽平, 曹明楠, 赵夕岗, 段京莉. 临床药物治疗杂志, 2020, 18 (2), 87.

[7] 段戴平, 曾会应. 大学化学, 2021, 36 (3), 2004044.

[8] 刘刚, 张恒, 马荣, 宋其圣, 苑世领, 孙国翠. 大学化学, 2020, 35 (6), 53.

[9] 李子倩. 酒精浓度越高消毒效果越好吗? 河南科技报, 2020-02-07. 